\title{
Corrigendum: Ginsenoside Rb1 Enhances Atherosclerotic Plaque Stability by Improving Autophagy and Lipid Metabolism in Macrophage Foam Cells
}

\section{OPEN ACCESS}

Edited and reviewed by: Satish Kitambi,

Karolinska Institute (KI), Sweden

*Correspondence:

Huixia Lu

luhuixia@sdu.edu.cn Wenqiang Chen

chenwenqiang33@sina.com

Specialty section:

This article was submitted to

Experimental Pharmacology and Drug

Discovery,

a section of the journal

Frontiers in Pharmacology

Received: 11 December 2017 Accepted: 19 December 2017

Published: 09 January 2018

Citation:

Qiao L, Zhang X, Liu M, Liu X Dong $M$, Cheng J, Zhang $X$, Zhai $C$, Song Y, Lu H and Chen W (2018)

Corrigendum: Ginsenoside Rb1

Enhances Atherosclerotic Plaque Stability by Improving Autophagy and

Lipid Metabolism in Macrophage Foam Cells. Front. Pharmacol. 8:964. doi: 10.3389/fphar.2017.00964

\begin{abstract}
Lei Qiao ${ }^{1}$, Xue Zhang ${ }^{1,2}$, Minghao Liu ${ }^{1}$, Xiaoling Liu ${ }^{1}$, Mei Dong ${ }^{1}$, Jing Cheng ${ }^{1}$, Xinyu Zhang ${ }^{1}$, Chungang Zhai ${ }^{1}$, Yu Song ${ }^{1}$, Huixia $L^{1 *}{ }^{1 *}$ and Wenqiang Chen ${ }^{1 *}$

${ }^{1}$ The Key Laboratory of Cardiovascular Remodeling and Function Research, Chinese Ministry of Education and Chinese Ministry of Health; the State and Shandong Province Joint Key Laboratory of Translational Cardiovascular Medicine, Department of Cardiology, Qilu Hospital of Shandong University, Jinan, China, ${ }^{2}$ Department of Cardiac Uhrasonography, Binzhou People's Hospital, Binzhou, China
\end{abstract}

Keywords: ginsenoside Rb1, atherosclerosis, lipid accumulation, macrophage foam cells, autophagy

\section{A corrigendum on}

Ginsenoside Rb1 Enhances Atherosclerotic Plaque Stability by Improving Autophagy and Lipid Metabolism in Macrophage Foam Cells

by Qiao, L., Zhang, X., Liu, M., Liu, X., Dong, M., Cheng, J., et al. (2017). Front. Pharmacol. 8:727. doi: 10.3389/fphar.2017.00727

In the published article, there was an error in affiliation [1 and 2]. Instead of " 1 The Key Laboratory of Cardiovascular Remodeling and Function Research, Chinese Ministry of Education and Chinese Ministry of Health, Jinan, China, ${ }^{2}$ The State and Shandong Province Joint Key Laboratory of Translational Cardiovascular Medicine, Department of Cardiology, Qilu Hospital, Shandong University, Jinan, Shandong, China", it should be "1 The Key Laboratory of Cardiovascular Remodeling and Function Research, Chinese Ministry of Education and Chinese Ministry of Health; the State and Shandong Province Joint Key Laboratory of Translational Cardiovascular Medicine, Department of Cardiology, Qilu Hospital of Shandong University, Jinan, Shandong, China." The authors apologize for this error and state that this does not change the scientific conclusions of the article in any way.

The original article has been updated.

Conflict of Interest Statement: The authors declare that the research was conducted in the absence of any commercial or financial relationships that could be construed as a potential conflict of interest.

Copyright $\odot 2018$ Qiao, Zhang, Liu, Liu, Dong, Cheng, Zhang, Zhai, Song, Lu and Chen. This is an open-access article distributed under the terms of the Creative Commons Attribution License (CC BY). The use, distribution or reproduction in other forums is permitted, provided the original author(s) or licensor are credited and that the original publication in this journal is cited, in accordance with accepted academic practice. No use, distribution or reproduction is permitted which does not comply with these terms. 